\title{
Phyllotaxis: from patterns of organogenesis at the meristem to shoot architecture
}

\author{
Carlos S. Galvan-Ampudia ${ }^{1}$, Anaïs M. Chaumeret ${ }^{1}{ }^{1}$ Christophe Godin $^{2}$, Teva Vernoux ${ }^{1, *}$ \\ ${ }^{1}$ Laboratoire de Reproduction et Développement des plantes, CNRS, INRA, ENS Lyon, UCBL, \\ Université de Lyon, 46 Allée d'Italie, Lyon 69364 Cedex 7, France \\ ${ }^{2}$ Virtual Plants Plants INRIA/CIRAD/INRA Project Team, Montpellier, France \\ Correspondence: teva.vernoux@ens-lyon.fr
}

\section{Abstract}

The primary architecture of the aerial part of plants is controlled by the shoot apical meristem, a specialized tissue containing a stem cell niche. The iterative generation of new aerial organs, (leaves, lateral inflorescences and flowers) at the meristem follows regular patterns, called phyllotaxis. Phyllotaxis has long been proposed to self-organize from the combined action of growth and of inhibitory fields blocking organogenesis in the vicinity of existing organs in the meristem. In this review we will highlight how a combination of mathematical/computational modeling and experimental biology has demonstrated that the spatio-temporal distribution of the plant hormone auxin controls both organogenesis and the establishment of inhibitory fields. We will discuss recent advances showing that auxin likely acts through a combination of biochemical and mechanical regulatory mechanisms that control not only the pattern of organogenesis in the meristem but also post-meristematic growth, to shape the shoot.

Introduction: the importance of the dynamics of organogenesis at the shoot apical meristem in setting shoot primary architecture

Higher plants undergo an extensive phase of post-embryonic development during which the plant not only expands but also produces organs along the apical-basal axis, e.g. the leaves and flowers for the aerial part of plants. This iterative production of new structures is under the influence of both developmental and environmental factors and is controlled by specialized structures containing stem cell niches called meristems. Two apical meristems, the shoot and root apical meristems, are established during embryogenesis at the two ends of the apical-basal axis. Their activity controls growth and development of the shoot and root primary axes, and they are thus called primary 
meristems. The shoot apical meristem first produces segments of stem that bear lateral leaves (at the axil of which lateral stems can be generated) and can elongate or remain compact depending on the species: during this period it is called the vegetative meristem. A combination of endogenous and environmental signals, including photoperiod, temperature and stress ${ }^{1}$, then triggers a transition from a vegetative meristem to an inflorescence meristem (IM) that produces the flowers or, in some cases, a single terminal flower. Here we will review our current knowledge of how organogenesis at the shoot apical meristem (which will simply be designated "meristem" from now on) contributes to shaping the primary architecture of higher plants.

The meristem is a highly organized tissue made up of a central zone (CZ) at the very tip of the axis where the stem cells are located, a peripheral zone (PZ) where lateral organs are initiated and the rib zone (RZ), which is situated beneath the $C Z$ and contains the organizing center (OC), a group of cells that play a central role in stem cell maintenance (Figure 1a). In a reference frame centered at the top of the growing meristem, stem cell daughters are progressively displaced away from the $C Z$ through growth (eulerian viewpoint) and become competent to produce new lateral organs when they reach the PZ. In Angiosperms, the meristem is, in addition, organized as a multilayered tissue where the outermost L1 and L2 cells proliferate and differentiate in the PZ to produce all epidermal and sub-epidermal cells, while the L3 underneath provides cells for the less organized inner tissues.

The activity of the meristem requires a variety of transcription factors, and a large number of these regulators have been identified through genetic studies conducted over the last twenty years (for an extensive review $s^{2}{ }^{2}$ ). Here, we will summarize only briefly the main molecular mechanisms controlling the maintenance of the stem cell niche and of meristem activity, identified mostly in Arabidopsis thaliana. The homeodomain transcription factor SHOOT MERISTEMLESS (STM) is expressed in all meristematic cells and required for the specification of meristem identity from embryogenesis onwards ${ }^{3}$. Once established, integrity of the stem cell niche and maintenance of the meristem rely on the expression of another homeodomain transcription factor, WUSCHEL (WUS) ${ }^{4}$, which is expressed in, and specifies, the $O C^{5}$, while the stem cells are marked by the expression of the CLAVATA3 (CLV3) gene which encodes a small signaling peptide ${ }^{6}$. WUS protein has been shown to move from cell to cell up to the CZ where it activates CLV3 production. In turn, perception of CLV3 by a receptor complex containing the CLAVATA1 (CLV1) receptor-like kinase ${ }^{5}$ represses the expression of WUS in the L1 and L2 layers of the CZ. WUS has additionally been shown to repress the expression of several members of the type A ARABIDOPSIS RESPONSE REGULATOR (ARR) gene family, which negatively regulate signaling in response to the plant hormone cytokinin. It has been proposed that cytokinin is produced specifically in the $L 1$ and that its distribution in the meristem 
participates in positioning the WUS domain (and thus the $\mathrm{OC}$ ) in the meristem through a positive feedback of cytokinin on WUS expression ${ }^{7,8}$. Thus, a non-linear network involving WUS, CLV3 and cytokinins defines the position and size of the stem cell niche in the meristem. Other independent pathways have also been implicated in this process and are reviewed in ${ }^{9}$. Organ initiation then involves important changes in cell identity resulting from two complementary processes: the loss of meristematic identity and the acquisition of organ identity. The first involves the repression of meristem identity genes such as STM ${ }^{3}$. In parallel, expression of organ-specific regulators such as LEAFY - LFY - and AINTEGUMENTA -ANT- controls the identity ${ }^{10}$ and outgrowth ${ }^{11}$ of the primordium, whilst specific sets of genes define its boundary region and apical-basal (or adaxial-abaxial) symmetry, together with the future vascular tissue.

A key property of organogenesis at the meristem is that it follows regular spatio-temporal patterns that vary depending on plant species, and also sometimes on developmental stages, from simple alternate arrangements to more complex whorled and spiral patterns. The relative time between organ initiations (called the plastochron), and a tight control of the spatial position of new organs in relation to existing ones, establish these characteristic developmental patterns. Lateral organs produced at the meristem are then distributed along the stem axis through growth. The pattern of organogenesis at the meristem thus defines the primary geometry of the stem or phyllotaxis. Phyllotaxis is characterized by the number of organs inserted on a node (also called jugacy) and by the relative divergence angle between organs. The prevalent phyllotactic patterns in higher plants are the decussate, distichous, whorled and spiral patterns. We have illustrated these patterns in Figure $1 \mathrm{~b}$. As the spatio-temporal pattern of organogenesis at the meristem is the primary determinant of phyllotaxis, the term phyllotaxis is also often used to designate the patterns at the meristem and we will use it in this sense in this review. However, as we will see below, the geometry of organ distribution along the shoot axis does not always directly reflect the pattern of organogenesis at the meristem, as post-meristematic growth also contributes significantly to the establishment of shoot primary architecture. To avoid confusion and at the same time keep a simple terminology, we will therefore refer to the geometric arrangement of organs along the stem as shoot phyllotaxis from now on.

Amongst phyllotactic patterns, the spiral pattern is the most common (see http://www.math.smith.edu/phyllo/About/Classification.html). Spiral phyllotaxis has intriguing mathematical features in that consecutive primordia are positioned relative to one another with a divergence angle close to $137,5^{\circ}$, known as the golden angle (Figure 1c). This angle corresponds to the division of a circle in two parts such that the ratio between the length of the smaller and the 
greater segment is equal to the ratio between the length of the greater segment and the circumference of the whole circle. When viewing the shoot from the top, other spirals, called the parastichies, can be defined by connecting organs in contact or in visible proximity either clockwise or anticlockwise (Figure 1d). The numbers of parastichies in each orientation are generally two consecutive numbers of the Fibonacci series. These mathematical regularities have inspired a long history of multidisciplinary studies making the field of phyllotaxis one of the oldest interdisciplinary fields of study in biology ${ }^{12}$. In this review, we will provide the reader with an overview of the most recent advances on phyllotaxis in higher plants, focusing mostly on spiral phyllotaxis, as it has been by far the most studied phyllotactic pattern. We will discuss how both mathematical/computational modeling and experimental biology has led to major breakthroughs in our understanding of this developmental process (for further reading see also: ${ }^{12,13}$ ).

\section{Phyllotaxis as a self-organizing process: the inhibitory field concept}

As pointed out above, the mathematical regularities observed in shoot phyllotaxis stimulated the development of mathematical, computational and physical models from the end of the $19^{\text {th }}$ century onwards $^{12}$. The most widely accepted model for phyllotaxis proposes that spatio-temporal patterns of organ initiation emerge from a self-organizing process involving lateral inhibition that is mediated by inhibitory fields generated by lateral organs in the growing shoot apex.

Some of the earlier observations supporting the idea that inhibitory fields are involved in controlling phyllotaxis were made by Hofmeister who noticed that primordium initiation is a sequential process that occurs at the meristem periphery, in the largest space left by the existing organs ${ }^{14}$. This led him to suggest the existence of geometrical constraints that could be interpreted as physical inhibitory fields, and would thus drive phyllotaxis. Experimental disruption of phyllotaxis through surgical incisions near organs in the meristem led to shifts of organ initiation sites that supported

Hofmeister's hypothesis ${ }^{15}$. Remarkably, although a regular phyllotactic pattern was quickly recovered in these experiments, this was not obligatorily a restoration of the original pattern. For instance, diagonal dissection of a decussate meristem resulted in recovery on each half of the meristem but with a shift to a spiral phylotaxis ${ }^{16}$. These pioneering results were later confirmed using local laser ablations in order to minimize secondary effects arising from tissue injuries ${ }^{17}$. Taken together, these experiments suggested the existence of a self-organized and self-correcting system robustly driving the spatio-temporal patterning of lateral organ production. As lateral organs are not always in physical contact, it was proposed early on that the properties of phyllotaxis could rely on 
chemical inhibition (rather than physical effects) from preexisting primordia, which influences the positioning of new primordia ${ }^{18,19}$. This led to the view that inhibitory fields block initiation in the vicinity of existing organs, while growth ultimately allows the initiation of new primordia at spatial positions where the cumulated inhibitory effects are the lowest (Figure 2). In the 1990's, Douady and Couder recapitulated these results using computational models ${ }^{20}$. They studied the dynamic properties of an inhibitory field-based model in detail, and demonstrated that this provides a simple conceptual framework for understanding phyllotaxis. In particular, they showed that inhibitory fields can drive self-organization of phyllotactic patterns and produce most, if not all phyllotactic patterns observed in nature by varying a single control parameter called $\Gamma$. This parameter corresponds to the ratio of the radius of inhibitory fields produced by organs divided by the radius of the generative circle at the center of the meristem where organs are initiated, thus highlighting the key importance of meristem geometry in determining phyllotactic patterns. It is important to note that postulating a generative circle suggests that another type of inhibitory field ensures that organs can be initiated only at a certain distance from the center of the meristem. This abstract model and its variants (models with fields of a geometric, physical or chemical nature have been proposed over the years: see ${ }^{12},{ }^{21}$ for a more extensive review) thus predict that inhibitory fields generated by organs could lead to the emergence of phyllotactic patterns in the growing meristem.

\section{A central role for auxin spatio-temporal distribution in phyllotaxis: biology and models}

It is only in the past two decades that the molecular mechanisms that establish the inhibitory fields predicted by models have been identified. Starting from the identification of the PIN-FORMED (PIN1) gene that encodes an auxin efflux carrier ${ }^{22}$, the plant hormone auxin has emerged as the central regulator of phyllotaxis. The fundamental role of auxin as an orchestrator of phyllotaxis is illustrated by the phenotype of the pin 1 mutant, which produces characteristic needle-like inflorescence stems devoid of organs ${ }^{23}$. Several studies have shown that auxin is transported directionally toward incipient primordia where it accumulates and activates a transcriptional response, initiating organogenesis upon auxin sensing ${ }^{24-26}$. A dynamic network of PIN1 auxin efflux carriers, whose cellular polarity determines the direction of the auxin flux (mainly in the L1 layer), regulates the spatio-temporal distribution of auxin in cooperation with influx carriers ${ }^{24,25,27}$. Analysis of the PIN1 auxin efflux carrier network led also to the proposition that auxin transport could not only promote the accumulation of auxin at organ initiation sites but could also deplete auxin levels around organs, thus generating inhibitory fields ${ }^{24}$. Spatio-temporal analysis of the distribution of the auxin signaling biosensor DII-VENUS, a synthetic protein degraded directly in response to auxin, allowed the direct visualization of the auxin-based inhibitory fields. While DII-VENUS fluorescence is absent from organs due to high auxin levels, the inhibitory fields can be visualized as domains of high 
fluorescence surrounding primordia and appear to be progressively established ${ }^{26,28}$. Taken together, these data indicate that it is the depletion of an activator, auxin, rather than the diffusion/movement of a repressor, that establishes chemical inhibitory fields centered around organs. Note however that the distribution of DII-VENUS fluorescence demonstrates that auxin also accumulates at the center of the meristem ${ }^{26}$. Thus auxin distribution does not explain the inhibitory field in the center of the meristem postulated in models. We will come back to this point in the next section.

As a consequence of the above results, several studies have addressed the question of whether selforganization of the PIN1 network could establish the auxin-based inhibitory fields. While molecular details of the mechanisms controlling PIN1 polarities at the cellular level are well known and involve intracellular trafficking of the protein (for a review see ${ }^{29}$ ), how the dynamics of PIN1 polarity are controlled at the tissular level is still largely unknown. Several theoretical models have proposed cellbased hypotheses that can reproduce auxin distribution patterns similar to those observed experimentally (for a more extensive review see ${ }^{30}$ ). Concentration-based models propose that PIN1 in a given cell is polarized toward the neighboring cell with the highest concentration of auxin ${ }^{31-33}$, while flux-based or canalization models (originally developed to reproduce vascular patterns) propose that a cell senses and enhances its own efflux of auxin, consequently stabilizing auxin flux between cells ${ }^{34-36}$. A combined model integrating both the concentration-based hypothesis in the L1 layer and the flux-based hypothesis in the vascular tissue was similarly shown to reproduce realistic PIN1 polarization dynamics in different developmental contexts including the meristem ${ }^{37}$. This last model recently received experimental support from the analysis of the localization and dynamics of PIN1 homologs in the meristem of Brachypodium distachyon ${ }^{38}$. The closest homologs of PIN1, BdPIN1a and BdPIN1b, are found in the developing vasculature of organs while a more distant homolog is found specifically in the L1 layer. These biological observations together with a combined model similar to the one developed by Bayer et al. $(2009)^{37}$ support the idea that PIN1 polarities could be controlled by different mechanisms in the epidermis layer and in the developing vasculature. Taken together, the different models indicate that polarization of PIN proteins controlled by a feedback between auxin and its own transport could provide self-organizing properties to the PIN1 auxin efflux carrier network in the meristem, and thus control phyllotaxis. However, the question of which type of feedback mechanism links auxin to its own efflux currently remains unanswered. Indeed a comparison of the properties of most of the models that have been published suggests that none can fully explain the dynamics of PIN1 during development ${ }^{39}$, highlighting the need for further knowledge of the molecular mechanisms underlying PIN1 
polarization in tissues. Here, modeling is again useful in identifying the molecular mechanisms that could be at play. For example, the work of Wabnik et al. $(2010)^{40}$ shows, using a detailed molecular model, that the modulation of PIN intracellular trafficking by an extracellular auxin receptor, together with a positive feedback of auxin on PIN transcription, could lead to PIN polarization as seen during vascular tissue development in leaves or during vascular tissue regeneration, two biological contexts that can be explained using the canalization hypothesis ${ }^{35,41}$. Consolidating our understanding of auxin signaling mechanisms and of the cellular mechanisms controlling PIN1 polarities in parallel with the development of mechanistic models will thus be instrumental for gaining a full understanding of how the PIN1 network drives phyllotaxis in the meristem.

\section{Gene regulatory networks regulating phyllotaxis}

Despite the differences in phyllotaxis between species, common regulatory gene networks determine maintenance and patterning of the meristem ${ }^{2,42}$. Some important players in these networks have been found to act in phyllotaxis through the regulation of auxin signaling, biosynthesis or transport, providing insights on how meristematic networks regulate phyllotaxis.

As discussed above, production of new organs in the meristem is initiated through accumulation of auxin at specific sites in the PZ. Auxin perception and signaling are controlled by a complex nonlinear pathway, involving nuclear-localized TIR1/AFB F-box co-receptors that are part of an SCF E3 ubiquitin ligase complex, and Aux/IAA transcriptional repressors. Auxin acts as a molecular glue to directly promote the interaction between TIR1/AFBs and Aux/IAAs and thus trigger polyubiquitination and degradation of Aux/IAAs ${ }^{43,44}$. At low auxin concentration, Aux/IAAs interact with the Auxin Response Factors (ARF) transcription factors. Both Aux/IAAs and ARFs are encoded by multigene families, comprising 29 and 23 members respectively in Arabidopsis. ARFs can be divided into two classes; either transcriptional activators (ARF5, ARF6, ARF7, ARF8 and ARF19 in Arabidopsis) or transcriptional repressors ${ }^{45}$. By promoting Aux/IAA degradation, auxin allows ARFs to modulate target gene transcription. Interactions between Aux/IAAs and ARFs are thus central to the regulation of auxin signaling. A combination of a large-scale analysis of Aux/IAA-ARF interactions, an analysis of the expression patterns of both gene families, and mathematical modeling of the pathway, has suggested that: 1- a differential expression of ARFs and Aux/IAAs between the CZ and the PZ creates a differential capacity to sense auxin between the two domains, the CZ being largely insensitive to auxin; 2- co-expression of ARF repressors and activators throughout the meristem gives buffering properties to the auxin signaling pathway and ensures robust transcriptional activation in organs (and thus organogenesis) ${ }^{26}$. Experimental support for these two predictions was obtained by comparing the spatio-temporal distribution of the DII-VENUS auxin biosensor to estimate auxin 
distribution and of the DR5::VENUS auxin-inducible synthetic reporter to monitor auxin-induced transcription ${ }^{26}$. While DII-VENUS indicates that auxin accumulates at the center of the meristem (as pointed out earlier), this does not induce transcription. In addition, auxin concentrations were found to vary significantly over time, while DR5::VENUS suggests that this does not induce fluctuations in auxin-induced transcription. Importantly, these results also indicate that a spatial regulation of the capacity to respond to auxin provides at least a partial molecular explanation for the absence of organ initiation at the center of the meristem. This suggests that a regulation of the sensitivity of cells to auxin provides the basis for the inhibitory field at the center of the meristem proposed in models ${ }^{20}$.

The AUXIN RESPONSE FACTOR 5/MONOPTEROS (ARF5/MP) is a master regulator of organ formation in the meristem ${ }^{46}$. Disruption of ARF5 function in the Arabidopsis meristem leads to the production of needle-like inflorescences similar to those of pin1 mutants, a phenotype that illustrates the key role of auxin signaling in the PZ. ARF5 was recently shown to directly activate the expression of the LFY, ANT, AINTEGUMENTA-LIKE6/PLETHORA3 (AIL6/PLT3) and FILAMENTOUS FLOWER (FIL) genes that are all essential regulators of flower development ${ }^{47,48}$. This study provides a molecular demonstration that auxin directly activates the transcriptional program leading to organ development (in this case the flower), as was previously indicated by the observation that local application of auxin at the $\mathrm{PZ}$ of pin 1 meristems triggers flower initiation ${ }^{49}$ and that $L F Y$ expression is down-regulated in the pin 1 mutant $^{50}$. This further supports an instructive role for auxin accumulation in triggering organogenesis and thus in phyllotaxis.

PLT genes encode members of the AP2-domain transcription factor family and are essential throughout plant development ${ }^{51}$. In the Arabidopsis meristem, three members of this family (PLT3, $P L T 5$ and $P L T 7$ ) are expressed in the CZ and PZ and are required for spiral phyllotaxis, as double or triple loss-of function mutants show an increased frequency of distichous phyllotactic patterns ${ }^{51}$. The expression of two flavin-containing monooxygenases, YUCCA1 (YUC1) and YUC4, which act in a ratelimiting step of auxin biosynthesis ${ }^{52}$, is reduced in plt3p/t5p/t7 triple mutants. Mutation of both YUC1 and YUC4 was also shown to induce strong perturbations in flower development ${ }^{53}$ and PIN1 expression is down-regulated in plt3plt5p/t7 mutants. Taken together, these observations suggest that PLTS act in a gene regulatory network that controls the abundance of auxin in the meristem through the regulation of auxin biosynthesis ${ }^{54}$. These data further point to a potential key role of auxin biosynthesis in phyllotaxis, a role that deserves consideration both in future biological experiments and phyllotaxis models. 


\section{Geometry of the meristem and phyllotaxis}

As mentioned in the first section, inhibitory field models highlight the importance of meristem geometry in setting the phyllotactic pattern (the $\Gamma$ parameter from the seminal work of Douady and Couder $\left.^{20}\right)$. Very few mutants with clear changes in the phyllotactic regime exist but these can likely be explained by a change in the geometry of the meristem. In maize, mutants impaired in the ABERRANT PHYLLOTAXIS 1 (ABPH1) protein, a two-component response regulator regulating cytokinin signaling, has a decussate rather than alternate phyllotaxis. This phenotype was correlated with a larger meristem while the size of lateral organs, the leaves in this case, appeared to be unchanged ${ }^{55,56}$. This observation is coherent with the well-established function of cytokinin in regulating the size of the stem cell niche (see introduction). As the abph1 mutation also affects PIN1 expression, the explanation for the phyllotactic phenotype could however be more complex and not linked solely to the change in the geometry of the meristem ${ }^{56}$. Another maize mutant, abph2, presents the same phenotype as abph1 and is caused by transposition of the gutaredoxin-encoding MALE STERILE CONVERTED ANTHER 1 (MSCA1) gene to a novel genomic location ${ }^{57}$. This transposition causes ectopic expression of MSCA1 and an enlargement of the meristem as seen in abph1. The MSCA1 protein interacts directly with FASCIATED EAR4 (FEA4), a bZIP transcription factor homologous to PERIANTHIA from Arabidopsis and that has been proposed to act in parallel with the WUS/CLV pathway in the regulation of meristem size ${ }^{58}$. This suggests that MSCA1 could regulate meristem size and in turn phyllotaxis in abph2 through modulating the activity of FEA4. In rice, decussate (dec) mutants might be also be disturbed in cytokinin signaling, although the molecular basis of this phenomenon remains unclear ${ }^{59}$. Again, dec mutants show a larger meristem and a decussate instead of an alternate phyllotaxis. The shared phyllotactic phenotype of the two abph mutants and the dec mutant further supports the fact that changes in meristem size in the mutants might be the primary trigger for the change in phyllotaxis, although this remains to be directly demonstrated.

\section{Post-meristematic growth also contributes to shoot phyllotaxis}

While the spatio-temporal pattern of organ initiation in the meristem is the primary level of control of shoot phyllotaxis, post-meristematic growth also makes an important contribution and several transgenic plants and mutants illustrate this. Ectopic expression of the boundary gene CUC was shown to have no effect on phyllotaxis in the meristem while inducing drastic changes in shoot phyllotaxis resulting in whorls of organ on the inflorescence stem ${ }^{60,61}$. The cuc2cuc3 double mutant also has an altered shoot phyllotaxis without major defects in the meristem (but see section 6). In 
these plants, growth and cell divisions patterns are modified in the internode on the stem, suggesting that an altered internode development could be the primary explanation for the shoot phyllotaxis phenotype in the cuc2cuc3 mutant ${ }^{62}$. Similarly the bellringer mutation leads to reduced cell expansion in internodes due to defective pectin methyl esterification ${ }^{63}$ and to alterations of the shoot phyllotactic pattern, with a clear tendency to form organ clusters on the stem. Taken together, these studies identify internode specification and elongation as a key developmental step in establishing a given shoot phyllotaxis.

A striking example of the contribution of post-meristematic growth to shoot phyllotaxis was also recently provided by the analysis of the cesa interactive protein 1 (csi1) mutant ${ }^{64}$. CSI1 acts in the regulation of growth by directly connecting the cortical microtubules to Cellulose Synthase complexes (CESA). The csi1 mutant presents a novel bimodal shoot phyllotaxis that is not seen in nature, in which plants have either a dominant phyllotactic angle of $90^{\circ}$ or of $180^{\circ}$ on the inflorescence stem. While phyllotaxis at the meristem is unchanged in the mutant, the mutation results in a slight torsion of the inflorescence stem. The authors demonstrated using a simple mathematical model that this torsion, combined with the fact that the ratio of internode length over stem diameter is rather invariant along the inflorescence axis, leads to one or the other dominant angles depending on whether the spiral at the meristem is left- or right-handed (which happens in equal proportions). The csi1 phyllotaxis phenotype thus demonstrates that post-meristematic growth can produce completely novel shoot phyllotaxis patterns, further highlighting the importance of post-meristematic growth regulation in shoot phyllotaxis.

\section{Temporal precision of organogenesis and phyllotaxis}

As pointed out already in the introduction, shoot phyllotaxis is often considered to provide a direct readout of spatio-temporal patterning at the shoot apical meristem despite the contribution of postmeristematic growth (section 5). In addition, the inhibitory field models we have discussed have contributed to a very regular and deterministic view of phyllotaxis, with organ initiations occurring sequentially at specific spatial positions. If this simplistic view were correct, the determination of the relative angles between organs in the meristem would indeed directly explain the relative angles found between fully developed organs on the stem. However, recent work shows that the situation is more complex at least for spiral phyllotaxis. Arabidopsis mutants in the gene encoding the cytokinin signaling inhibitor ARABIDOPSIS HISTIDINE PHOSPHOTRANSFER PROTEIN 6 (AHP6) were found to have characteristic defects in shoot phyllotaxis that motivated a careful analysis of the dynamics of organ initiation at the shoot apical meristem using live-imaging ${ }^{65}$. This demonstrated 
that while relative angle specification in wild-type meristems is extremely robust, the plastochron is on the contrary very plastic, resulting frequently in very short or null plastochrons and thus to organ co-initiations. The frequency of organ co-initiations was significantly increased in ahp6 mutant meristems without any detectable effects on the spatial positioning of organs, thus identifying AHP6 as a specific regulator of the robustness of the plastochron at the meristem. AHP6 was, in addition, shown to act as a moving signal in the meristem ${ }^{65,66}$. AHP6 is expressed specifically in organs early after their initiation. The expression of AHP6 is regulated by auxin and the AHP6 proteins moves to create inhibitory fields of cytokinin signaling. The movement of AHP6 creates a differential in AHP6 levels and in cytokinin signaling activity between the site where the new organ is being produced and that where the next organ initiation event is expected. The differential in AHP6 concentration provides positional cues that promote sequential initiation of organs, explaining the plastochron noise-filtering function of AHP6. As mentioned above, shoot phyllotaxis of the ahp6 mutant clearly deviates from that of wild-type plants (when analyzing the inflorescence), due to an increase in the frequency of defects that are nonetheless also observed, albeit at lower frequencies, in wild-type plants ${ }^{65,67}$. Indeed an analysis of both wild-type and ahp6 shoot phyllotaxis demonstrated deviations from the canonical Fibonacci spiral that can be explained if the position of several consecutive organs along the stem is permuted (in comparison with the canonical distribution) without affecting the angular positioning of organs. These deviations were thus called permutations. The frequency of permutations is significantly increased in ahp6 mutants, suggesting that co-initiations of organs at the meristem result in the permutations observed on the inflorescence shoot axis. A plausible interpretation of this phenomenon, supported by an extensive statistical analysis of shoot phyllotaxis $^{67}$ and a theoretical analysis of the effect of noise on inhibitory fields models ${ }^{68}$, is that internodes are established even when organs are co-initiated. However, the development of the internode distributes co-initiated organs along the stem randomly (Figure 3). This idea is supported by the fact that 1 ) the size of internodes is significantly smaller when organs are permuted and that, 2) the frequency of organ co-initiation events in the meristem is twice the frequency of permutations observed on the inflorescence stem ${ }^{65,67}$. These studies thus identify noise on the plastochron as a genetically-controlled phenomenon that, combined with post-meristematic growth (internode development), directly affects the robustness of shoot phyllotaxis by causing deviations of the relative angle between organs from the expected golden angle. Of course this work also highlights a key role for cytokinin in regulating phyllotaxis downstream of auxin.

Interestingly, the occurrence of co-initiations and permutations was also found to change in different Arabidopsis accessions or mutants and with environmental conditions (when testing different light regimes ${ }^{64}$ ). This revealed a correlation between meristem size and shoot phyllotaxis 
robustness. Indeed, the conditions and genotypes tested showed variations in meristem sizes indicating that lower levels of organ permutations and co-initiations might result from a decrease in meristem size (without apparent changes in organ size). These results again highlight the importance of meristem geometry for phyllotaxis, but in this case the change in geometry is not sufficient to significantly modify the phyllotactic pattern. Instead it appears to affect the coupling between the spatial positioning of organs and the timing of their initiation. These observations also indicate that the noise in the plastochron is sensitive to environmental conditions. Finally, it has been proposed that the abnormal phyllotaxis of the cuc2cuc3 mutant that we discussed in section 5 , despite being largely due to post-meristematic growth defects, could also result in part from an increase in organ permutations ${ }^{62}$. This suggests that organ co-initiation at the meristem could be buffered by complex gene networks implicating AHP6 as well as the CUC genes.

\section{A role for mechanical forces in phyllotaxis?}

Until now we have addressed only chemical and molecular players involved in phyllotaxis. However a role for mechanical signals in phyllotaxis has also been proposed. Plant cells are under turgor pressure and are physically attached to their neighbors by cell walls. Geometry, together with growth, can create dynamic fields of mechanical forces in the meristem that can be either tensile or compressive ${ }^{69,70}$. Such forces could act downstream of chemical signals controlling morphogenesis but could also, in theory, be instructive for developmental patterning in the meristem and thus act in parallel with chemical signals such as auxin. To correlate mechanical forces and meristem function, Paul Green and co-workers developed a biophysical model in which primordium initiation was considered to be the result of compressive forces in the epidermis, a view fueled by a large body of previous modeling work (more discussion can be found in ${ }^{69}$ ). Differential growth between internal tissues and the epidermis was proposed to generate compressive stresses in the epidermis resulting from pushing forces. These lead to deformation of the epidermis, a phenomenon called buckling, and to outgrowth of the organs ${ }^{71}$. However, while compressive forces can be observed in the concave meristems of certain species such as the sunflower ${ }^{71}$, meristematic tissues are generally convex and likely to be under tension (i.e. exposed to pulling forces). The actual contribution of buckling in organ initiation thus remains to be demonstrated, although it could in theory act cooperatively with auxin-based mechanisms to drive phyllotaxis ${ }^{69}$.

More recently a collection of studies has revealed that local changes in mechanical properties are intrinsically associated with organ outgrowth and suggested ways in which this might impact meristem activity and phyllotaxis. Auxin has long been known to induce a reduction in apoplastic 
$\mathrm{pH}$, which in turn causes cell wall softening ${ }^{72}$, supporting the idea that auxin could trigger changes in the mechanical properties of tissues. Changes in tissues mechanical properties could also be mediated by Pectin Methyl-Esterases (PMEs), which target the major cell wall component pectin, and have been shown to be necessary for cell wall loosening during organ initiation and for subsequent organ outgrowth ${ }^{73-75}$. The expression of PME5 is controlled by the homeodomain transcription factor BELLRINGER (BLR) ${ }^{63}$, mutations in which induce important defects in phyllotaxis. The phyllotactic defects of blr mutants are in part due to defects in internode elongation, thus providing another example of the importance of post-meristematic growth in phyllotaxis (section 5). However, BLR also acts to exclude PME5 from the meristem proper, thus restricting the expression of PME5, and thus rapid growth, to organs ${ }^{63}$. Conversely, inhibition of pectin methyl-esterification due to over-expression of the PME Inhibitor PMEI3 leads to the production of pin-shaped meristems, whilst ectopic application of PME to the meristem leads to perturbations in phyllotactic patterning 73. In addition, immuno-labeling experiments have confirmed that pectins are de-methyl-esterified during organ initiation ${ }^{73}$. Taken together, these studies demonstrate that a dynamic regulation of cell wall composition likely plays an important role not only during post-meristematic growth, but also at the meristem where it might be essential in establishing patterns of organogenesis. This view is further supported by several independent approaches using modeling and direct measurements of the mechanical properties of the meristem that demonstrate that the $C Z$ is stiffer than the $P Z{ }^{76,77}$. These differential mechanical properties, which closely match the differential sensitivity of cells to auxin $^{26}$, could thus restrict growth in the center of the meristem and allow for organ outgrowth at the periphery.

It has also recently been demonstrated that microtubules align preferentially with the main direction of mechanical stress in the meristem. This observation led to the proposal that microtubules might sense mechanical stress (through an unknown mechanism) and guide anisotropic deposition of cellulose, thus counteracting the mechanical stress ${ }^{78,79}$. Mechanical stress could also have a direct impact on auxin distribution as PIN1 efflux transporters have been shown to localize preferentially to membranes that are oriented tangentially to the direction of growth imposed by microtubule orientation ${ }^{80}$. A partial coupling between PIN1 localization and microtubule orientation could then create a feedback from growth-driven mechanical forces on auxin fluxes, contributing to the robustness of phyllotaxis. The extensive interplay between auxin and mechanics in the meristem is further illustrated by a recent study that demonstrated, using both biological experiments and modeling, that auxin accumulation triggers a shift from an anisotropic to an isotropic distribution of microtubules in cells at sites of organ initiation ${ }^{81}$. Together with cell wall softening mediated by cell- 
wall modifying enzymes, this is thought to permit local changes in growth orientation allowing organ emergence in response to auxin. Taken together, these different studies support a scenario in which phyllotaxis is driven by auxin through the coordinated action of both genetic and biochemical pathways and of mechanical forces at the meristem. These factors feedback, in turn, onto auxin distribution dynamics.

\section{Conclusion}

While our understanding of phyllotaxis still remains partial, notably due to the fact that few phyllotactic mutants have been identified, recent years have seen tremendous advances that have identified the plant hormone auxin as the major regulator of phyllotaxis. A role for mechanical feedbacks in phyllotaxis is also emerging, providing an interesting model system in which to analyze how chemical and mechanical signal cooperate to control morphogenesis. Modeling has been crucial in these advances and provides a rich toolbox for understanding how the mechanisms identified could explain the self-organizing properties of this unique developmental system. The emergence of powerful live-imaging approaches has also been instrumental in the analysis of the dynamic properties of the phyllotactic system, revealing the importance of the timing of organ initiation in controlling shoot phyllotaxis. The development of an auxin biosensor has also allowed the visualization of the auxin-based inhibitory fields, and opened the possibility of further analyzing how these fields are formed. Modeling has also suggested that temporal variations in the strength of the fields might be important for the stability of phyllotaxis ${ }^{31,32}$. The development of mechanical sensors will be an important next step in order to provide precise information regarding the spatio-temporal distribution of mechanical forces in the meristem. Combining such quantitative approaches with molecular genetics (for example by identifying the mechanosensors acting in the meristem) may provide key experimental data that, coupled with further refinement of the existing models, should push forward our understanding of phyllotaxis, notably by clarifying the relative contribution of chemical and mechanical signals. Finally, it will also be important to question whether current knowledge of mechanisms regulating spiral phyllotaxis is fully relevant to all types of phyllotaxis including whorled and multijugate modes, or whether other mechanisms are involved.

\section{Acknowledgments}

We thank Gwyneth Ingram for critical reading of the manuscript. Research in the authors' laboratory is supported by the HFSP Research Grant RPG 054-2013 (to CGA, TV \& CG), the Inria project lab 
Morphogenetics (to $\mathrm{CG}$ ) and by the Institut de Biologie Computationelle (to $\mathrm{CG}$ ) and by a predoctoral fellowship from the French Ministry of Research (to AC).

\section{References}

1. Andres F, Coupland G. The genetic basis of flowering responses to seasonal cues. Nat Rev Genet. 2012;13(9):627-639. doi:10.1038/nrg3291.

2. Barton MK. Twenty years on: the inner workings of the shoot apical meristem, a developmental dynamo. Dev Biol. 2010;341(1):95-113. doi:10.1016/j.ydbio.2009.11.029.

3. Long JA, Moan El, Medford JI, Barton MK. A member of the KNOTTED class of homeodomain proteins encoded by the STM gene of Arabidopsis. Nature. 1996;379(6560):66-69. doi:10.1038/379066a0.

4. Gordon SP, Chickarmane VS, Ohno C, Meyerowitz EM. Multiple feedback loops through cytokinin signaling control stem cell number within the Arabidopsis shoot meristem. Proc Natl Acad Sci U S A. 2009;106(38):16529-16534. doi:10.1073/pnas.0908122106.

5. Yadav RK, Perales M, Gruel J, Girke T, Jonsson H, Reddy GV. WUSCHEL protein movement mediates stem cell homeostasis in the Arabidopsis shoot apex. Genes Dev. 2011;25(19):2025-2030. doi:10.1101/gad.17258511.

6. Fletcher JC, Brand U, Running MP, Simon R, Meyerowitz EM. Signaling of cell fate decisions by CLAVATA3 in Arabidopsis shoot meristems. Science. 1999;283(5409):1911-1914.

7. Shani E, Yanai O, Ori N. The role of hormones in shoot apical meristem function. Curr Opin Plant Biol. 2006;9(5):484-489. doi:10.1016/j.pbi.2006.07.008.

8. Chickarmane VS, Gordon SP, Tarr PT, Heisler MG, Meyerowitz EM. Cytokinin signaling as a positional cue for patterning the apical-basal axis of the growing Arabidopsis shoot meristem. Proc Natl Acad Sci U S A. 2012;109(10):4002-4007. doi:10.1073/pnas.1200636109.

9. Heidstra R, Sabatini S. Plant and animal stem cells: similar yet different. Nat Rev Mol Cell Biol. 2014;15(5):301-312. doi:10.1038/nrm3790.

10. Parcy F, Nilsson O, Busch MA, Lee I, Weigel D. A genetic framework for floral patterning. Nature. 1998;395(6702):561-566. doi:10.1038/26903. 
11. Mizukami Y, Fischer RL. Plant organ size control: AINTEGUMENTA regulates growth and cell numbers during organogenesis. Proc Natl Acad Sci U S A. 2000;97(2):942-947.

12. Adler I, Barabe D, Jean RV. A History of the Study of Phyllotaxis. Ann Bot. 1997;80(3):231244. doi:10.1006/anbo.1997.0422.

13. Kuhlemeier C. Phyllotaxis. Trends Plant Sci. 2007;12(4):143-150.

doi:10.1016/j.tplants.2007.03.004.

14. Hofmeister W. Allgemeine Morphologie der Gewächse. In: Handbuch Der Physiologischen Botanik I-2. Leipzig: W. Engelmann; 1868.

15. Snow M, Snow R. Experiments on Phyllotaxis. I. The Effect of Isolating a Primordium. Philos Trans R Soc Lond Ser B Contain Pap Biol Character. 1931;221(ArticleType: research-article / Full publication date: 1932 / Copyright (C) 1932 The Royal Society):1-43. doi:10.2307/92206.

16. Snow M, Snow R. Experiments on Phyllotaxis. Part III. Diagonal Splits through Decussate Apices. Philos Trans R Soc Lond B Biol Sci. 1935;225(519):63-94. doi:10.2307/92254.

17. Reinhardt $\mathrm{D}$, Frenz $\mathrm{M}$, Mandel $\mathrm{T}$, Kuhlemeier $\mathrm{C}$. Microsurgical and laser ablation analysis of leaf positioning and dorsoventral patterning in tomato. Dev Camb Engl. 2005;132(1). doi:10.1242/dev.01544.

18. Wardlaw CW. Further Experimental Observations on the Shoot Apex of Dryopteris aristata Druce. Philos Trans R Soc Lond B Biol Sci. 1949;233(604):415-451. doi:10.1098/rstb.1949.0001.

19. Richards FJ. Phyllotaxis: Its Quantitative Expression and Relation to Growth in the Apex. Philos Trans R Soc Lond B Biol Sci. 1951;235(629):509-564. doi:10.1098/rstb.1951.0007.

20. Douady S, Couder Y. Phyllotaxis as a Dynamical Self Organizing Process Part II: The Spontaneous Formation of a Periodicity and the Coexistence of Spiral and Whorled Patterns. J Theor Biol. 1996;178(3):275-294. doi:10.1006/jtbi.1996.0025.

21. Shipman PD, Newell a. C. Polygonal planforms and phyllotaxis on plants. J Theor Biol. 2005;236:154-197. doi:10.1016/j.jtbi.2005.03.007.

22. Galweiler L, Guan C, Muller A, Wisman E, Mendgen K, Yephremov A, Palme K. Regulation of polar auxin transport by AtPIN1 in Arabidopsis vascular tissue. Science. 1998;282(5397):2226-2230. 
23. Okada K, Ueda J, Komaki MK, Bell CJ, Shimura Y. Requirement of the Auxin Polar Transport System in Early Stages of Arabidopsis Floral Bud Formation. Plant Cell. 1991;3(7). doi:10.1105/tpc.3.7.677.

24. Reinhardt D, Pesce E-R, Stieger P, Mandel T, Baltensperger K, Bennett M, Traas J, Friml J, Kuhlemeier C. Regulation of phyllotaxis by polar auxin transport. Nature. 2003;426(6964):255-260. doi:10.1038/nature02081.

25. Heisler MG, Ohno C, Das P, Sieber P, Reddy GV, Long JA, Meyerowitz EM. Patterns of auxin transport and gene expression during primordium development revealed by live imaging of the Arabidopsis inflorescence meristem. Curr Biol CB. 2005;15(21):1899-1911. doi:10.1016/j.cub.2005.09.052.

26. Vernoux T, Brunoud G, Farcot E, Morin V, Van den Daele H, Legrand J, Oliva M, Das P, Larrieu $A$, Wells $D$, et al. The auxin signalling network translates dynamic input into robust patterning at the shoot apex. Mol Syst Biol. 2011;7. doi:10.1038/msb.2011.39.

27. Bainbridge K, Guyomarc'h S, Bayer E, Swarup R, Bennett M, Mandel T, Kuhlemeier C. Auxin influx carriers stabilize phyllotactic patterning. Genes Dev. 2008;22(6):810-823. doi:10.1101/gad.462608.

28. Brunoud G, Wells DM, Oliva M, Larrieu A, Mirabet V, Burrow AH, Beeckman T, Kepinski S, Traas J, Bennett MJ, et al. A novel sensor to map auxin response and distribution at high spatiotemporal resolution. Nature. 2012;482(7383):103-106. doi:10.1038/nature10791.

29. Friml J. Subcellular trafficking of PIN auxin efflux carriers in auxin transport. Eur J Cell Biol. 2010;89(2-3):231-235. doi:10.1016/j.ejcb.2009.11.003.

30. Wabnik K, Kleine-Vehn J, Govaerts W, Friml J. Prototype cell-to-cell auxin transport mechanism by intracellular auxin compartmentalization. Trends Plant Sci. 2011;16(9):468-475. doi:10.1016/j.tplants.2011.05.002.

31. Smith RS, Guyomarc'h S, Mandel T, Reinhardt D, Kuhlemeier C, Prusinkiewicz P. A plausible model of phyllotaxis. Proc Natl Acad Sci U S A. 2006;103(5):1301-1306. doi:10.1073/pnas.0510457103.

32. Smith RS, Kuhlemeier C, Prusinkiewicz P. Inhibition fields for phyllotactic pattern formation: a simulation studyThis article is one of a selection of papers published on the Special Theme of Shoot Apical Meristems. Can J Bot. 2006;84(11):1635-1649. doi:10.1139/b06-133. 
33. Jonsson H, Heisler MG, Shapiro BE, Meyerowitz EM, Mjolsness E. An auxin-driven polarized transport model for phyllotaxis. Proc Natl Acad Sci U S A. 2006;103(5):1633-1638. doi:10.1073/pnas.0509839103.

34. Mitchison GJ, Hanke DE, Sheldrake AR. The Polar Transport of Auxin and Vein Patterns in Plants [and Discussion]. Philos Trans R Soc Lond B Biol Sci. 1981;295(1078):461-471. doi:10.1098/rstb.1981.0154.

35. Rolland-Lagan A-G, Prusinkiewicz P. Reviewing models of auxin canalization in the context of leaf vein pattern formation in Arabidopsis. Plant J Cell Mol Biol. 2005;44(5):854-865. doi:10.1111/j.1365-313X.2005.02581.x.

36. Stoma S, Lucas M, Chopard J, Schaedel M, Traas J, Godin C. Flux-based transport enhancement as a plausible unifying mechanism for auxin transport in meristem development. PLoS Comput Biol. 2008;4(10). doi:10.1371/journal.pcbi.1000207.

37. Bayer EM, Smith RS, Mandel T, Nakayama N, Sauer M, Prusinkiewicz P, Kuhlemeier C. Integration of transport-based models for phyllotaxis and midvein formation. Genes Dev. 2009;23(3):373-384. doi:10.1101/gad.497009.

38. O'Connor DL, Runions A, Sluis A, Bragg J, Vogel JP, Prusinkiewicz P, Hake S. A Division in PINMediated Auxin Patterning during Organ Initiation in Grasses. PLoS Comput Biol. 2014;10(1):e1003447. doi:10.1371/journal.pcbi.1003447.

39. van Berkel K, de Boer RJ, Scheres B, ten Tusscher K. Polar auxin transport: models and mechanisms. Dev Camb Engl. 2013;140(11):2253-2268. doi:10.1242/dev.079111.

40. Wabnik K, Kleine-Vehn J, Balla J, Sauer M, Naramoto S, Reinöhl V, Merks RMH, Govaerts W, Friml J. Emergence of tissue polarization from synergy of intracellular and extracellular auxin signaling. Mol Syst Biol. 2010;6:447-447. doi:10.1038/msb.2010.103.

41. Sauer M, Balla J, Luschnig C, Wisniewska J, Reinohl V, Friml J, Benkova E. Canalization of auxin flow by Aux/IAA-ARF-dependent feedback regulation of PIN polarity. Genes Dev. 2006;20(20):2902-2911. doi:10.1101/gad.390806.

42. Bartlett ME, Thompson B. Meristem identity and phyllotaxis in inflorescence development. Front Plant Sci. 2014;5. doi:10.3389/fpls.2014.00508.

43. Dharmasiri N, Dharmasiri S, Estelle M. The F-box protein TIR1 is an auxin receptor. Nature. 2005;435(7041):441-445. doi:10.1038/nature03543. 
44. Kepinski S, Leyser O. The Arabidopsis F-box protein TIR1 is an auxin receptor. Nature. 2005;435(7041):446-451. doi:10.1038/nature03542.

45. Guilfoyle TJ, Hagen G. Auxin response factors. Curr Opin Plant Biol. 2007;10(5):453-460. doi:10.1016/j.pbi.2007.08.014.

46. Zhao Z, Andersen SU, Ljung K, Dolezal K, Miotk A, Schultheiss SJ, Lohmann JU. Hormonal control of the shoot stem-cell niche. Nature. 2010;465(7301):1089-1092. doi:10.1038/nature09126.

47. Yamaguchi N, Yamaguchi A, Abe M, Wagner D, Komeda Y. LEAFY controls Arabidopsis pedicel length and orientation by affecting adaxial-abaxial cell fate. Plant J. 2012;69:844-856. doi:10.1111/j.1365-313X.2011.04836.x.

48. Wu M-F, Yamaguchi N, Xiao J, Bargmann B, Estelle M, Sang Y, Wagner D. Auxin-regulated chromatin switch directs acquisition of flower primordium founder fate. eLife. 2015;4. doi:10.7554/eLife.09269.

49. Reinhardt $D$, Mandel $T$, Kuhlemeier $C$. Auxin regulates the initiation and radial position of plant lateral organs. Plant Cell. 2000;12(4):507-518.

50. Vernoux T, Kronenberger J, Grandjean O, Laufs P, Traas J. PIN-FORMED 1 regulates cell fate at the periphery of the shoot apical meristem. Dev Camb Engl. 2000;127(23):5157-5165.

51. Prasad K, Grigg SP, Barkoulas M, Yadav RK, Sanchez-Perez GF, Pinon V, Blilou I, Hofhuis H, Dhonukshe P, Galinha C, et al. Arabidopsis PLETHORA transcription factors control phyllotaxis. Curr Biol. 2011;21:1123-1128. doi:10.1016/j.cub.2011.05.009.

52. Zhao Y, Christensen SK, Fankhauser C, Cashman JR, Cohen JD, Weigel D, Chory J. A role for flavin monooxygenase-like enzymes in auxin biosynthesis. Science. 2001;291(5502):306-309. doi:10.1126/science.291.5502.306.

53. Cheng $Y$, Dai $X$, Zhao Y. Auxin synthesized by the YUCCA flavin monooxygenases is essential for embryogenesis and leaf formation in Arabidopsis. Plant Cell. 2007;19(8):2430-2439. doi:10.1105/tpc.107.053009.

54. Pinon V, Prasad K, Grigg SP, Sanchez-Perez GF, Scheres B. Local auxin biosynthesis regulation by PLETHORA transcription factors controls phyllotaxis in Arabidopsis. Proc Natl Acad Sci U S A. 2013;110(24):1107-1112. doi:10.1073/pnas.1213497110. 
55. Giulini A, Wang J, Jackson D. Control of phyllotaxy by the cytokinin-inducible response regulator homologue ABPHYL1. Nature. 2004;430:1031-1034. doi:10.1038/nature02778.

56. Lee B, Johnston R, Yang Y, Gallavotti A, Kojima M, Travencolo BAN, Costa L da F, Sakakibara $H$, Jackson D. Studies of aberrant phyllotaxy1 mutants of maize indicate complex interactions between auxin and cytokinin signaling in the shoot apical meristem. Plant Physiol. 2009;150(1):205216. doi:10.1104/pp.109.137034.

57. Yang F, Bui HT, Pautler M, Llaca V, Johnston R, Lee B, Kolbe A, Sakai H, Jackson D. A maize glutaredoxin gene, abphyl2, regulates shoot meristem size and phyllotaxy. Plant Cell. 2015;27(1):121-131. doi:10.1105/tpc.114.130393.

58. Pautler M, Eveland AL, LaRue T, Yang F, Weeks R, Lunde C, Je BI, Meeley R, Komatsu M, Vollbrecht E, et al. FASCIATED EAR4 encodes a bZIP transcription factor that regulates shoot meristem size in maize. Plant Cell. 2015;27(1):104-120. doi:10.1105/tpc.114.132506.

59. Itoh Jl, Hibara KI, Kojima M, Sakakibara H, Nagato Y. Rice DECUSSATE controls phyllotaxy by affecting the cytokinin signaling pathway. Plant J. 2012;72:869-881. doi:10.1111/j.1365313x.2012.05123.x.

60. Peaucelle A, Laufs P. Phyllotaxy: Beyond the Meristem and Auxin Comes the miRNA. Plant Signal Behav. 2007;2(May 2015):293-295. doi:10.4161/psb.2.4.4040.

61. Sieber P, Wellmer F, Gheyselinck J, Riechmann JL, Meyerowitz EM. Redundancy and specialization among plant microRNAs: role of the MIR164 family in developmental robustness. Dev Camb Engl. 2007;134(6):1051-1060. doi:10.1242/dev.02817.

62. Burian a., Raczy ska-Szajgin M, Borowska-Wykr t D, Piatek A, Aida M, Kwiatkowska D. The CUP-SHAPED COTYLEDON2 and 3 genes have a post-meristematic effect on Arabidopsis thaliana phyllotaxis. Ann Bot. 2015:807-820. doi:10.1093/aob/mcv013.

63. Peaucelle A, Louvet R, Johansen JN, Salsac F, Morin H, Fournet F, Belcram K, Gillet F, Höfte H, Laufs $P$, et al. The transcription factor BELLRINGER modulates phyllotaxis by regulating the expression of a pectin methylesterase in Arabidopsis. 2011;4741:4733-4741. doi:10.1242/dev.072496.

64. Landrein B, Refahi Y, Besnard F, Hervieux N, Mirabet V, Boudaoud A, Vernoux T, Hamant O. Meristem size contributes to the robustness of phyllotaxis in Arabidopsis. J Exp Bot. 2015;66(5):1317-1324. doi:10.1093/jxb/eru482. 
65. Besnard F, Refahi Y, Morin V, Marteaux B, Brunoud G, Chambrier P, Rozier F, Mirabet V, Legrand J, Laine S, et al. Cytokinin signalling inhibitory fields provide robustness to phyllotaxis. Nature. 2014;505(7483):417-421. doi:10.1038/nature12791.

66. Besnard F, Rozier F, Vernoux T. The AHP6 cytokinin signaling inhibitor mediates an auxincytokinin crosstalk that regulates the timing of organ initiation at the shoot apical meristem. Plant Signal Behav. 2014;9(4).

67. Guedon Y, Refahi Y, Besnard F, Farcot E, Godin C, Vernoux T. Pattern identification and characterization reveal permutations of organs as a key genetically controlled property of postmeristematic phyllotaxis. J Theor Biol. 2013;338. doi:10.1016/j.jtbi.2013.07.026.

68. Mirabet $\mathrm{V}$, Besnard $\mathrm{F}$, Vernoux $\mathrm{T}$, Boudaoud A. Noise and robustness in phyllotaxis. PLoS Comput Biol. 2012;8(2). doi:10.1371/journal.pcbi.1002389.

69. Newell AC, Shipman PD, Sun Z. Phyllotaxis as an example of the symbiosis of mechanical forces and biochemical processes in living tissue. Plant Signal Behav. 2008;3(8):586-589.

70. Robinson S, Burian A, Couturier E, Landrein B, Louveaux M, Neumann ED, Peaucelle A, Weber A, Nakayama N. Mechanical control of morphogenesis at the shoot apex. J Exp Bot. 2013;64(15):4729-4744. doi:10.1093/jxb/ert199.

71. Green PB. Expression of pattern in plants: combining molecular and calculus-based biophysical paradigms. Am J Bot. 1999;86(8):1059-1076.

72. Jacobs M, Ray PM. Rapid Auxin-induced Decrease in Free Space pH and Its Relationship to Auxin-induced Growth in Maize and Pea. Plant Physiol. 1976;58(2):203-209.

73. Peaucelle A, Louvet R, Johansen JN, Hofte H, Laufs P, Pelloux J, Mouille G. Arabidopsis phyllotaxis is controlled by the methyl-esterification status of cell-wall pectins. Curr Biol CB. 2008;18(24):1943-1948. doi:10.1016/j.cub.2008.10.065.

74. Peaucelle A, Braybrook SA, Le Guillou L, Bron E, Kuhlemeier C, Hofte H. Pectin-induced changes in cell wall mechanics underlie organ initiation in Arabidopsis. Curr Biol CB. 2011;21(20):1720-1726. doi:10.1016/j.cub.2011.08.057.

75. Braybrook SA, Peaucelle A. Mechano-chemical aspects of organ formation in Arabidopsis thaliana: the relationship between auxin and pectin. PloS One. 2013;8(3). doi:10.1371/journal.pone.0057813. 
76. Milani P, Gholamirad M, Traas J, Arnéodo A, Boudaoud A, Argoul F, Hamant O. In vivo analysis of local wall stiffness at the shoot apical meristem in Arabidopsis using atomic force microscopy. Plant J. 2011;67(6):1116-1123. doi:10.1111/j.1365-313X.2011.04649.x.

77. Kierzkowski D, Nakayama N, Routier-Kierzkowska A-L, Weber A, Bayer E, Schorderet M, Reinhardt D, Kuhlemeier C, Smith RS. Elastic domains regulate growth and organogenesis in the plant shoot apical meristem. Science. 2012;335(6072):1096-1099. doi:10.1126/science.1213100.

78. Hamant $\mathrm{O}$, Heisler MG, Jonsson $\mathrm{H}$, Krupinski $\mathrm{P}$, Uyttewaal $M$, Bokov $\mathrm{P}$, Corson $F$, Sahlin $\mathrm{P}$, Boudaoud A, Meyerowitz EM, et al. Developmental patterning by mechanical signals in Arabidopsis. Science. 2008;322(5908):1650-1655. doi:10.1126/science.1165594.

79. Nakayama N, Smith RS, Mandel T, Robinson S, Kimura S, Boudaoud A, Kuhlemeier C. Mechanical regulation of auxin-mediated growth. Curr Biol CB. 2012;22(16):1468-1476. doi:10.1016/j.cub.2012.06.050.

80. Heisler MG, Hamant O, Krupinski P, Uyttewaal M, Ohno C, Jonsson H, Traas J, Meyerowitz EM. Alignment between PIN1 polarity and microtubule orientation in the shoot apical meristem reveals a tight coupling between morphogenesis and auxin transport. PLOS Biol. 2010;8(10). doi:10.1371/journal.pbio.1000516.

81. Sassi M, Ali O, Boudon F, Cloarec G, Abad U, Cellier C, Chen X, Gilles B, Milani P, Friml J, et al. An auxin-mediated shift toward growth isotropy promotes organ formation at the shoot meristem in Arabidopsis. Curr Biol CB. 2014;24(19):2335-2342. doi:10.1016/j.cub.2014.08.036. 
A

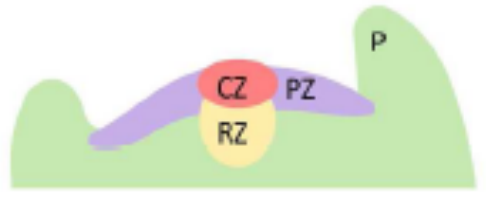

B
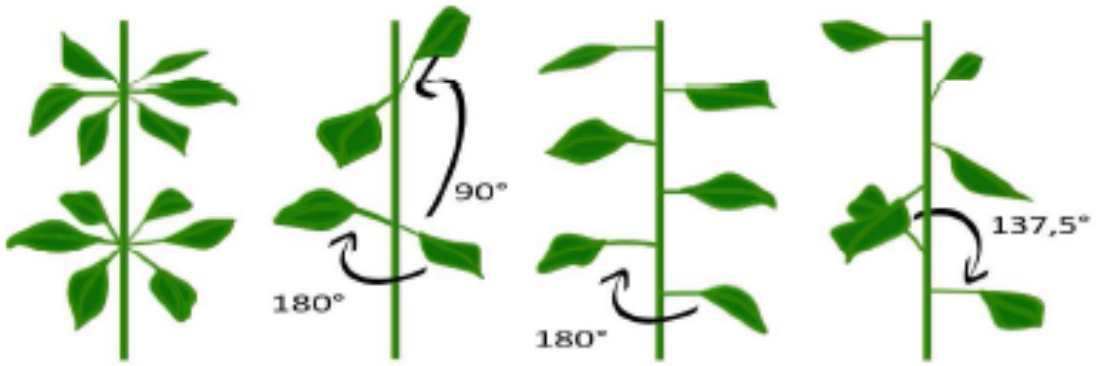

C

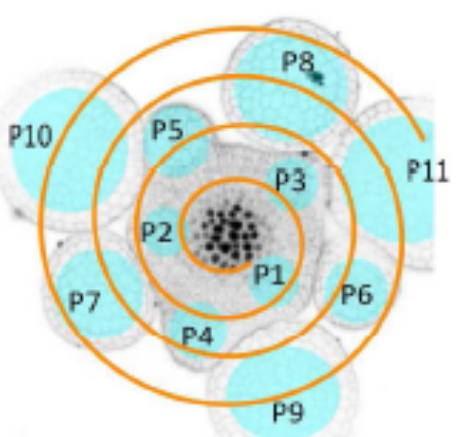

$\bar{D}$

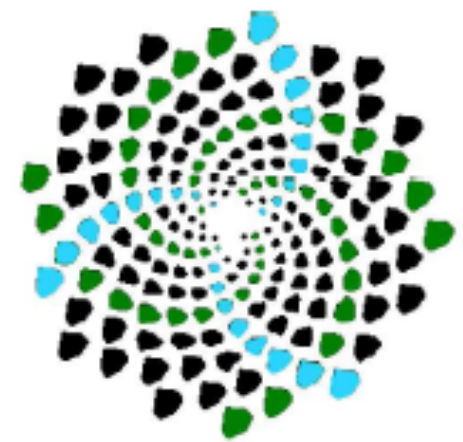

Figure 1: Meristem organization and phyllotaxis. (A) Meristem functional organization. The meristem is composed of three distinct functional zones: the central zone (CZ) that contains the stem cells, the surrounding peripheral zone (PZ) from which the primordia $(P)$ arise and the rib zone (RZ) where is found the organizing center establishing the stem cell niche. (B) Common patterns of phyllotaxis. From left to right: whorled (with several organs at each node), opposite-decussate (with successive pairs of opposite organs at $90^{\circ}$ ), distichous with a divergence angle of $180^{\circ}$ between successive organs, and Fibonacci spiral (with a divergence angle of $137,5^{\circ}$ between successive organs). (C) Top view of the inflorescence of Arabidopsis thaliana showing how primordia follow a Fibonacci spiral. (D) Contact parastichies: connecting each organ to its closest neighbors creates clockwise and anti-clockwise spirals called contact parastichies. On this example, 13 clockwise contact parastichies (several of them are highlighted in green) and 21 anti-clockwise contact 
parastichies (several of them are highlighted in blue) can be found. 13 and 21 are two consecutive numbers of the Fibonacci series. This image was modified from https://www.mathsisfun.com/numbers/nature-golden-ratio-fibonacci.html

P5

i1

P2

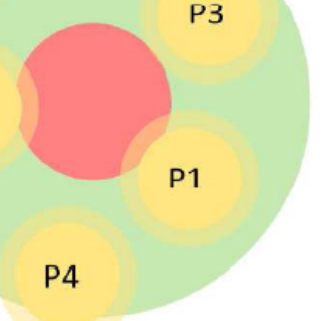

P6

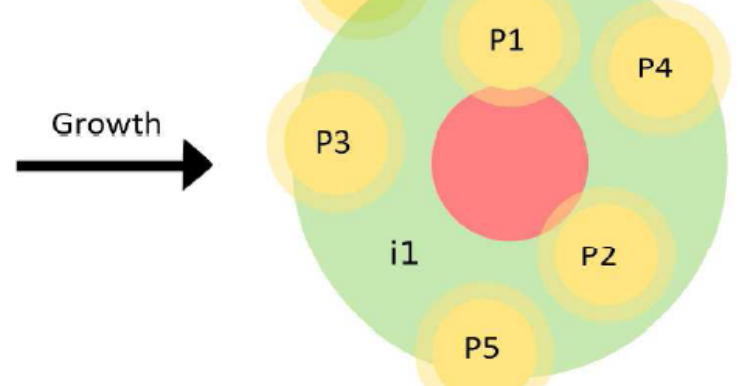

Figure 2: Inhibitory fields and phyllotaxis. Existing primordia (numbered from the youngest, P1, to the oldest, P5) generate inhibitory fields that block organ initiation in their vicinity. In this example (a spiral phyllotaxis), growth moves the existing organs away from the tip of the meristem, thus lowering the inhibition and allowing for the next initiation to occur (i1). This process repeats itself as the plant grows. 
A

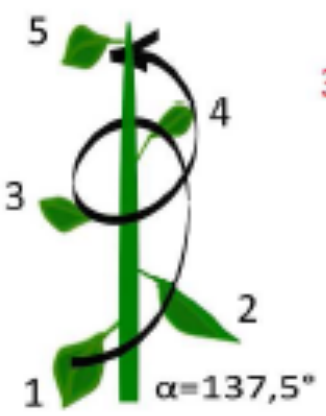

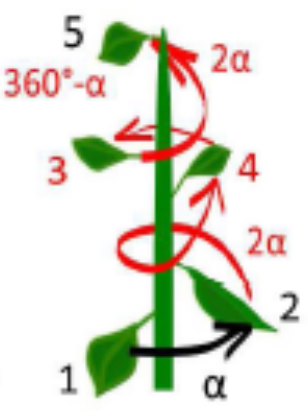

B

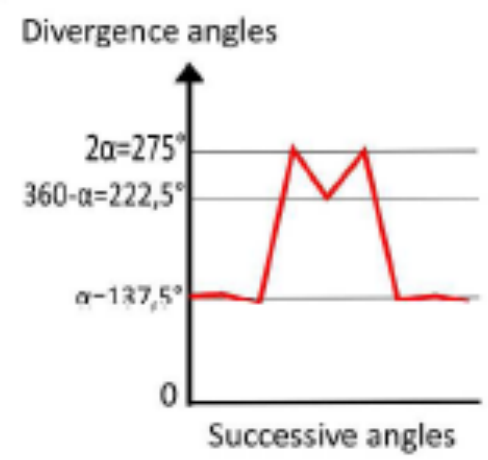

Figure 3: Permutations in spiral phyllotaxis. As discussed in the main text, two organs that are initiated simultaneously at the meristem can lead either to a normal organ distribution or to a permutation of the order of organs along the stem, depending on which organ is positioned above the other after the development of the internode. (A) A canonical sequence of divergence angles along a stem with a spiral phyllotaxis (left) and the same sequence but with a permutation of organs 3 and 4 (right). (B) A single permutation gives rise to a new angle sequence: $2 \alpha, 360-\alpha, 2 \alpha$. (with $\left.\alpha=137^{\circ}\right)$. 